ORIGINAL ARTICLE

\title{
Involvement of MMP-7 in invasion of pancreatic cancer cells through activation of the EGFR mediated MEK-ERK signal transduction pathway
}

\author{
X Tan, H Egami, M Abe, F Nozawa, M Hirota, M Ogawa
}

J Clin Pathol 2005;58:1242-1248. doi: 10.1136/jcp.2004.025338

See end of article for authors' affiliations

....................

Correspondence to: Dr H Egami, Department of Gastroenterological

Surgery, Graduate School of Medical Sciences, Kumamoto University, Honjo 1-1-1, Kumamoto 860-8556, Japan; tanxd@ hotmail.com

Accepted for publication 6 April 2005

\begin{abstract}
Aims: To clarify the involvement of matrix metalloproteinase-7 (MMP-7) in cell dissociation and the subsequent invasion of pancreatic cancer cells.

Methods: Western blotting, in vitro invasion assay, immunocytochemistry, and immunohistochemistry were performed in pancreatic cancer cell lines or pancreatic cancer tissue.

Results: The active form of the MMP-7 protein was expressed exclusively in the conditioned medium of dissociated (PC-1.0 and AsPC-1) pancreatic cancer cells, whereas proMMP-7 protein was only detected in the conditioned medium of non-dissociated (PC-1 and Capan-2) cells. Both intracellular and conditioned medium localised MMP-7 was greatly reduced by treatment with the epidermal growth factor receptor (EGFR) inhibitor AG1478 and the mitogen activated protein kinase kinase (MEK) inhibitor U0126 in pancreatic cancer cells. MMP-7 treatment significantly induced the disruption of tight junction (TJ) structures and subsequent cell dissociation, and activation of the EGFR mediated MEK- ERK (extracellular signal regulated protein kinase) signalling pathway in the non-dissociated pancreatic cancer cells. Moreover, the strong in vitro invasiveness of dissociated cells was inhibited by AG1478 and U0126 treatment, whereas the weak invasiveness of non-dissociated cells was apparently induced by MMP-7 treatment. In addition, MMP-7 expression was stronger at the invasive front than at the centre of human pancreatic tumours.

Conclusion: MMP-7 is involved in cell dissociation and the subsequent invasion of pancreatic cancer cells. It induces the disruption of TJ structures and forms a positive feedback loop with activation of the EGFR mediated MEK-ERK signalling pathway.
\end{abstract}

A the time of diagnosis, pancreatic cancer usually shows extensive local invasion and/or metastasis, precluding a curative surgical resection. Cancer cell dissociation has been shown to be the first and pivotal step in the invasionmetastasis process. ${ }^{1}$ However, the cellular and molecular mechanisms of invasion and metastasis in pancreatic cancer have not been fully elucidated.

\begin{abstract}
"Matrix metalloproteinase-7 has been implicated in tumour invasion-metastasis and in tumour initiation or growth in gastrointestinal and other cancers"
\end{abstract}

Two hamster pancreatic cancer cell lines with a different invasion-metastasis potential-the weakly invasive cell line PC-1 and the highly invasive cell line PC-1.0-were established previously. ${ }^{23}$ In our recent research, the epidermal growth factor receptor (EGFR) mediated mitogen activated protein kinase kinase 2 (MEK2)-ERK2 (extracellular signal regulated kinase 2) signal transduction pathway ${ }^{4-6}$ was implicated in cancer cell dissociation through disrupting the tight junction (TJ) structures in hamster and human dissociated (PC-1.0 and AsPC-1, respectively) and nondissociated (PC-1 and Capan-2, respectively) pancreatic cancer cell lines. ${ }^{7-9}$

In addition, matrix metalloproteinase-7 (MMP-7, matrilysin-1) has been implicated in tumour invasion-metastasis and in tumour initiation or growth in gastrointestinal and other cancers. ${ }^{10}$ Furthermore, positivity for MMP-7, rather than other MMP molecules, significantly correlates with the extent of tumour invasion, lymph node and distant metastasis, and advanced tumour stage in pancreatic carcinoma. ${ }^{11}$
However, because conventional assays are based on endpoint results, whether MMP-7 is involved in cell dissociation in pancreatic cancer remains unclear.

In our study, the involvement of MMP-7 in TJ disruption, activation of the EGFR mediated MEK-ERK signalling pathway, and the in vitro invasiveness of pancreatic cancer cells were examined to clarify the potential role of MMP-7 in the regulation of cell dissociation and subsequent invasion of pancreatic cancer cells

\section{MATERIALS AND METHODS}

\section{Cell lines and cell culture}

We used hamster dissociated (PC-1.0) and non-dissociated (PC-1) pancreatic cancer cell lines. The PC-1 cell line was established from pancreatic ductal/ductular adenocarcinoma induced by BOP ( $N$-nitrosobis(2-oxopropyl) amine) in a Syrian golden hamster. ${ }^{2}$ The PC- 1.0 cell line was established from a subcutaneous tumour produced after inoculation of PC-1 cells. ${ }^{3}$ The human dissociated (AsPC-1) and nondissociated (Capan-2) pancreatic cancer cell lines (American Tissue Culture Collection, Rockville, Maryland, USA) were also used. PC-1 and Capan-2 cells grow as island-like colonies, whereas PC-1.0 and AsPC-1 cells grow as single cells.

These cells were incubated in RPMI-1640 (Gibco-BRL, Grand Island, New York, USA), supplemented with 10\% fetal bovine serum (Bioserum, Victoria, Australia), 100 units $/ \mathrm{ml}$

\footnotetext{
Abbreviations: EGFR, epidermal growth factor receptor; ERK, extracellular signal regulated protein kinase; $\mathrm{FI}$, fluorescence intensity; MEK, mitogen activated protein kinase kinase; MMP-7, matrix metalloproteinase-7; p-, phosphorylated; TJ, tight junction; ZO-1, zonula occludens- 1
} 

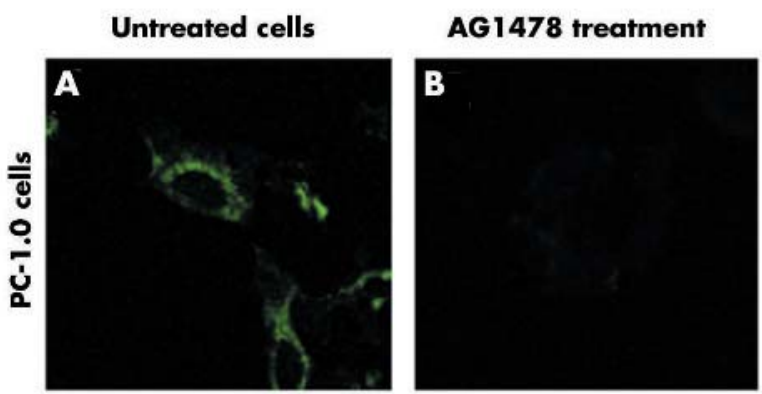

\section{U0126 treatment}
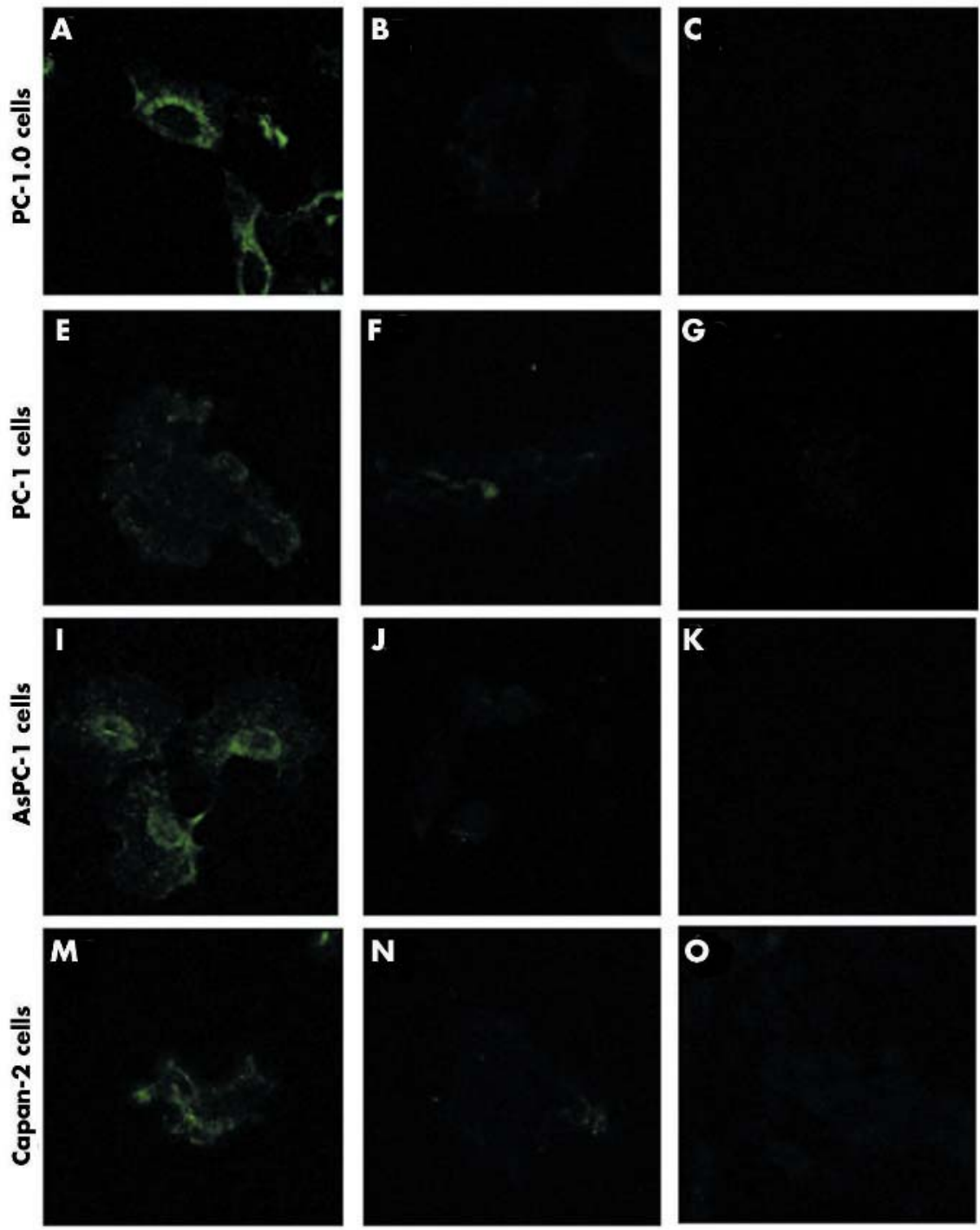
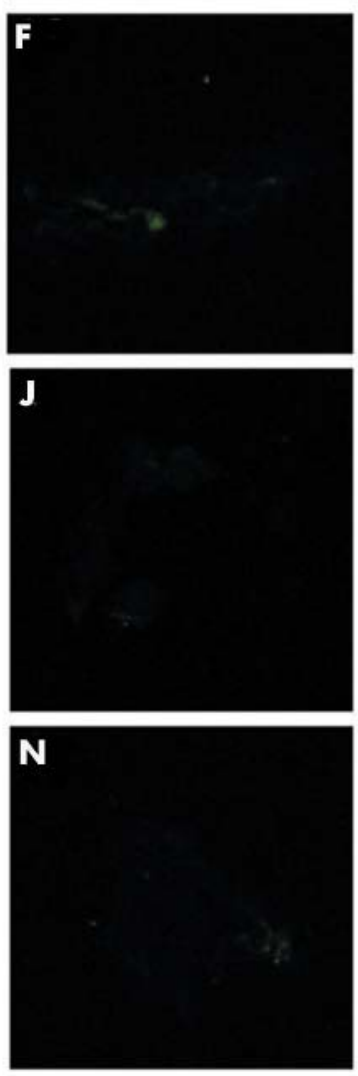
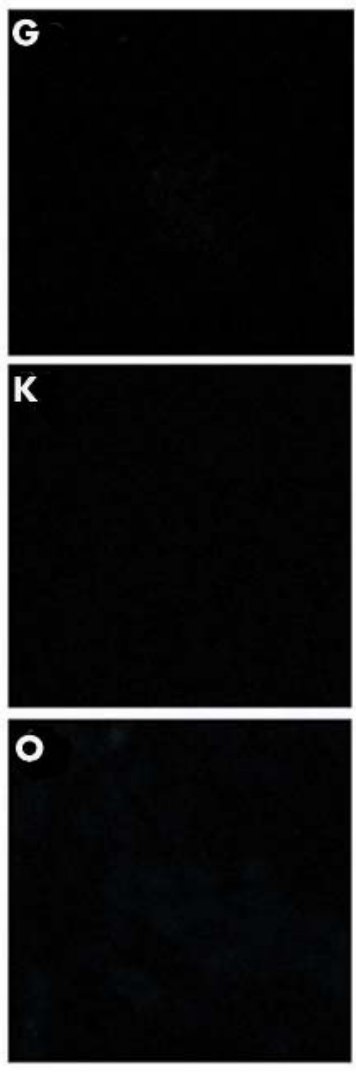

FI of MMP-7 expression
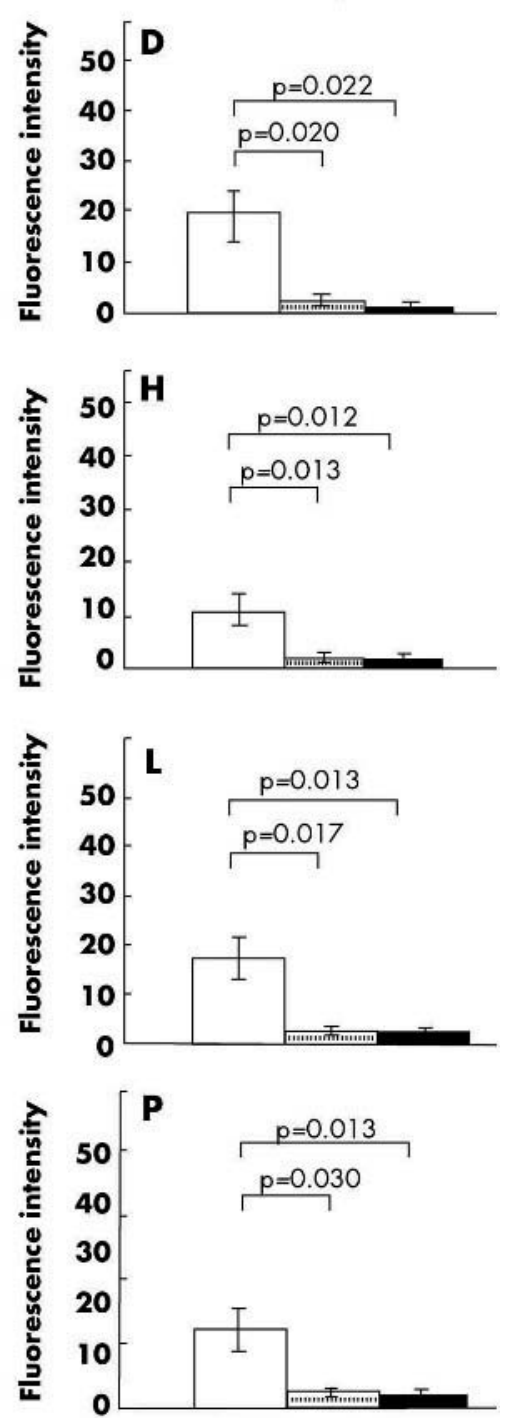

Figure 1 Immunofluorescent staining for matrix metalloproteinase-7 (MMP-7) and fluorescent intensity (FI) values in pancreatic cancer cells. Original magnification, $\times 400$. Open bars, untreated cells; shaded bars, AG1478; closed bars, U0126.

penicillin $\mathrm{G}$, and $100 \mu \mathrm{g} / \mathrm{ml}$ streptomycin at $37^{\circ} \mathrm{C}$ in a humidified atmosphere of $5 \% \mathrm{CO}_{2}$ to $95 \%$ air. The cells were serum starved overnight before experiments.

\section{Antibodies}

We used rabbit antihuman MMP-7 (Oncogene Research Products, San Diego, California, USA), occludin and zonula occludens-1 (ZO-1) (Zymed Laboratories Inc, South San Francisco, California, USA), phosphorylated EGFR (p-EGFR), phosphorylated MEK (p-MEK), and phosphorylated ERK ( $p$ ERK) (all Santa Cruz Biotechnology, Santa Cruz, California, USA) polyclonal antibodies in our study. These antibodies are crossreactive with hamster tissues. ${ }^{4-7} 9$ We also used fluorescein isothiocyanate and rhodamine labelled fluorescence secondary antibodies (Santa Cruz Biotechnology).

\section{Tissue samples}

We obtained 37 pancreatic cancer tissue samples at the time of surgery from the department of surgery II, Kumamoto University Hospital from October 1989 to July 2001. The median age of the patients with pancreatic cancer was 63.5 years (range, 35-78); there were 14 men and 23 women. Histologically, the samples consisted of 13 well differentiated, 20 moderately differentiated, and four poorly differentiated adenocarcinomas. All of the tissue samples were histologically examined, and the pathological diagnoses were confirmed.

\section{Preparation of cell lysate and concentration of conditioned medium}

We grew the four pancreatic cancer cell lines mentioned earlier in $90 \mathrm{~mm}$ dishes. After growing to subconfluent, we replaced the medium with serum free medium and incubated the cells for 36 hours with or without $10 \mu \mathrm{M}$ specific EGFR inhibitor AG1478 (Calbiochem, San Diego, California, USA) ${ }^{12}$ or $10 \mu \mathrm{M}$ specific MEK inhibitor U0126 (Cell Signaling Technology, Beverly, Massachusetts, USA). ${ }^{13}$

We then collected the conditioned medium and concentrated it 50 fold. Simultaneously, we lysed the cells using RIPA cell lysis buffer. ${ }^{8}$ We adjusted the concentration of each 

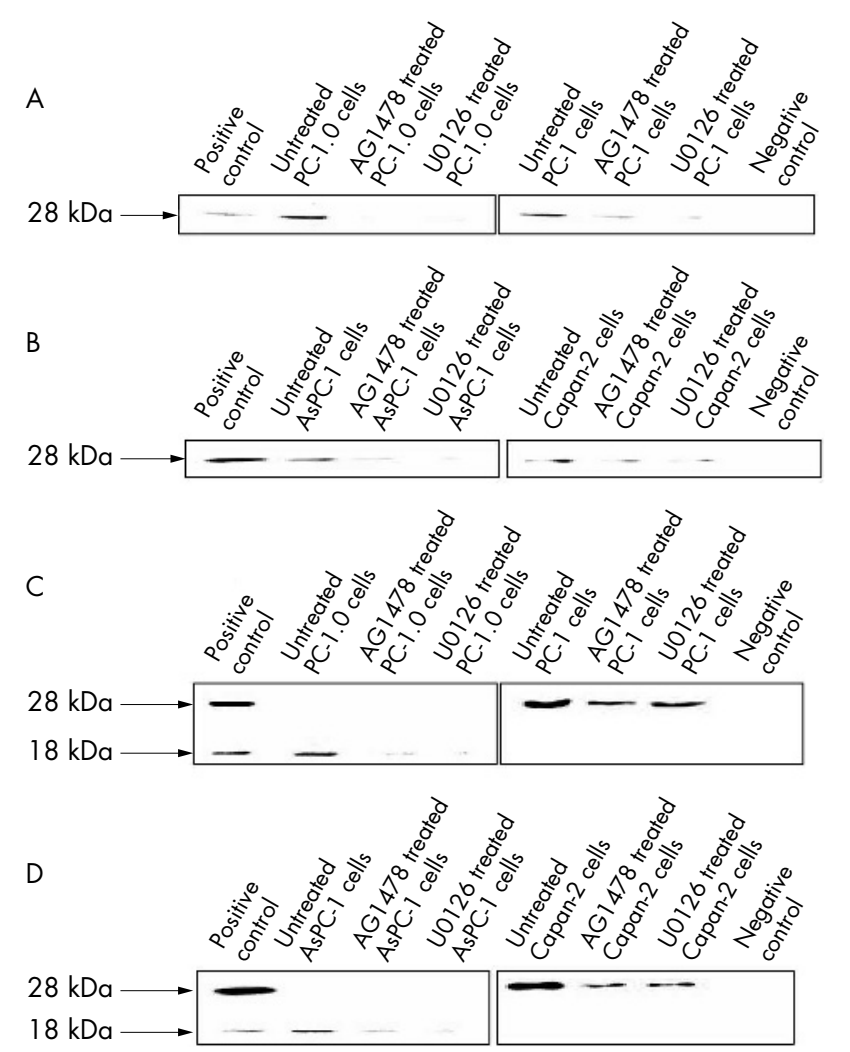

Figure 2 Western blotting for the matrix metalloproteinase-7 (MMP-7) protein in pancreatic cancer cells. The intracellular expression of MMP-7 was inhibited by AG1478 or U0126 treatment in (A) PC-1.0 and PC-1 cells, in addition to (B) AsPC-1 and Capan-2 cells. Inactive and active MMP-7 showed significantly different expression in the conditioned medium between dissociated and non-dissociated pancreatic cancer cells PC-1.0 and PC-1 (C), and AsPC-1 and Capan-2 (D). A lysate of SW620 cells and distilled water were used as positive and negative controls, respectively. The molecular weight is indicated.

sample to $1 \mathrm{mg} / \mathrm{ml}$ using the Bio-Rad protein assay kit (BioRad, Anaheim, California, USA).

\section{Western blot analysis}

We performed western blotting as described previously. ${ }^{8}$ In brief, we ran samples of equivalent total protein $(20 \mu \mathrm{g})$ on a $15 \%$ polyacrylamide slab gel. Then we incubated the membranes with primary antibodies overnight at $4{ }^{\circ} \mathrm{C}$, after which we incubated the blots with horseradish peroxidase conjugated secondary antibody. We used enhanced chemiluminescence (Santa Cruz Biotechnology) to detect the signals developed on Kodak scientific imaging film (Eastman Kodak Company, Rochester, New York, USA). We used a cell lysate of SW620 cells ${ }^{14}$ and distilled water as positive and negative controls, respectively.

\section{Immunofluorescent staining and fluorescence intensity analysis}

We planted the cells mentioned earlier on the chamber slides and incubated them before the experiment. For the activation study, we incubated the pancreatic cancer cells with $100 \mathrm{ng} /$ ml human purified MMP-7 (a mixture of zymogen and active enzyme; Sigma, St Louis, Missouri, USA) for 36 hours. For the inhibition study, we incubated the cells with $10 \mu \mathrm{M}$ AG1478 or $10 \mu \mathrm{M}$ U0126 for 36 hours.

After incubation, immunofluorescent staining was performed as described previously. ${ }^{4}$ We prepared the control slides as follows: (1) we processed sections without a primary antibody; (2) we used normal rabbit serum and non-specific rabbit IgG instead of a polyclonal antibody.

Finally, we chose six cells in the image randomly to measure the fluorescence intensity (FI) with the software Fluoview 500 (version 4.3; Olympus, Tokyo, Japan). We used the averages for FI analysis of the expression of MMP-7, pEGFR, p-MEK, and p-ERK.

\section{In vitro invasion assay}

We carried out the in vitro invasion assay using Invasion Chambers (Becton Dickinson Labware, Bedford, Massachusetts, USA) according to the method described previously. ${ }^{15}$ We incubated the cells for 12 hours at $37^{\circ} \mathrm{C}$ with or without $10 \mu \mathrm{M}$ AG1478, 10 $\mu \mathrm{M}$ U0126, or $100 \mathrm{ng} / \mathrm{ml}$ human MMP-7. We determined the number of cells migrating to the lower side of the filter by counting the number of nuclei within an area of $1 \mathrm{~mm}^{2}$ on the grid at a magnification of $\times 100$.

\section{Immunohistochemical analysis}

We performed immunohistochemical staining using the avidin-biotin-peroxidase complex technique (Vectastain Elite ABC kit; Vector Laboratories, Burlingame, California, USA), as described previously. ${ }^{4}$ We prepared the control slides as follows: (1) we processed sections without a primary antibody; (2) we used normal rabbit serum and non-specific rabbit IgG instead of a polyclonal anti-MMP-7 antibody.

\section{Statistical analysis}

We analysed the average FI for MMP-7, p-EGFR, p-MEK, and p-ERK in the different experimental groups, and the numbers of pancreatic cancer cells counted in the invasion assay, by the unpaired Student's $t$ test with the Stat View computerised program (SAS Institute Inc, Cary, North Carolina, USA). We considered $\mathrm{p}<0.05$ to be significant.

\section{RESULTS}

AG 1478 and U0126 downregulated both intracellular and conditioned medium localised expression of MMP-7 protein in pancreatic cancer cells

We found constitutive expression of MMP-7 protein in dissociated cells PC-1.0 and AsPC-1 (mean FI, 19.18 (SD, $5.20)$ and 17.27 (4.26), respectively; fig lA,I). MMP-7 expression was relatively weak in non-dissociated cells PC-1 and Capan-2 (mean FI, 11.51 (SD, 2.92) and 12.72 (3.54), respectively; fig $1 \mathrm{E}, \mathrm{M})$. Furthermore, MMP-7 expression was mainly found in the cytoplasm. The expression of MMP-7 was greatly reduced after treatment with AG1478 or U0126 in dissociated cells PC-1.0 (mean FI, 2.11 (SD, 0.92) and 1.28 (0.49), respectively; $\mathrm{p}=0.020$ and $\mathrm{p}=0.022$; fig $1 \mathrm{~B}, \mathrm{C}$ ) and AsPC-1 (mean FI, 2.26 (SD, 0.79) and 1.89 (1.25), respectively; $\mathrm{p}=0.017$ and $\mathrm{p}=0.013$; fig $1 \mathrm{~J}, \mathrm{~K})$, and in non-dissociated cells PC-1 (mean FI, 2.41 (SD, 1.07) and 2.38 (1.15), respectively; $p=0.013$ and $p=0.012$; fig $1 F, G)$ and Capan-2 (mean FI, 2.66 (SD, 0.43) and 1.65 (1.37), respectively; $\mathrm{p}=0.030$ and $\mathrm{p} 0.013$; fig $1 \mathrm{~N}, \mathrm{O}$ ). Figure $\mathrm{l}$ parts $\mathrm{D}, \mathrm{H}, \mathrm{L}$, and $\mathrm{P}$ show the average FI values for MMP-7 expression in the different pancreatic cancer cells.

The western blotting data confirmed the immunofluorescent staining results (fig 2A,B). Moreover, the western blotting data showed that the active form of the MMP-7 protein ( $17 \mathrm{kDa}$ in hamster cells and $18 \mathrm{kDa}$ in human cells) was detected only in the conditioned medium of dissociated pancreatic cancer cells PC-1.0 and AsPC-1 (fig 2C,D), whereas the inactive form of the MMP-7 protein $(27 \mathrm{kDa}$ in hamster cells and $28 \mathrm{kDa}$ in human cells) was found in the conditioned medium of untreated PC-1 and Capan-2 cells (fig 2C,D). In addition, both intracellular and conditioned medium localised MMP-7 proteins were reduced by 
PC-1 cells
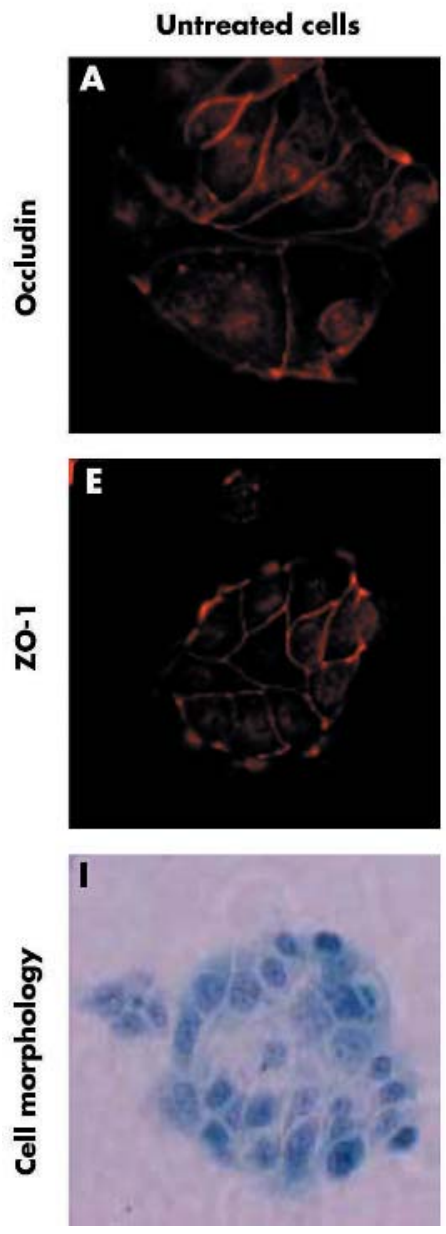

MMP-7 treatment
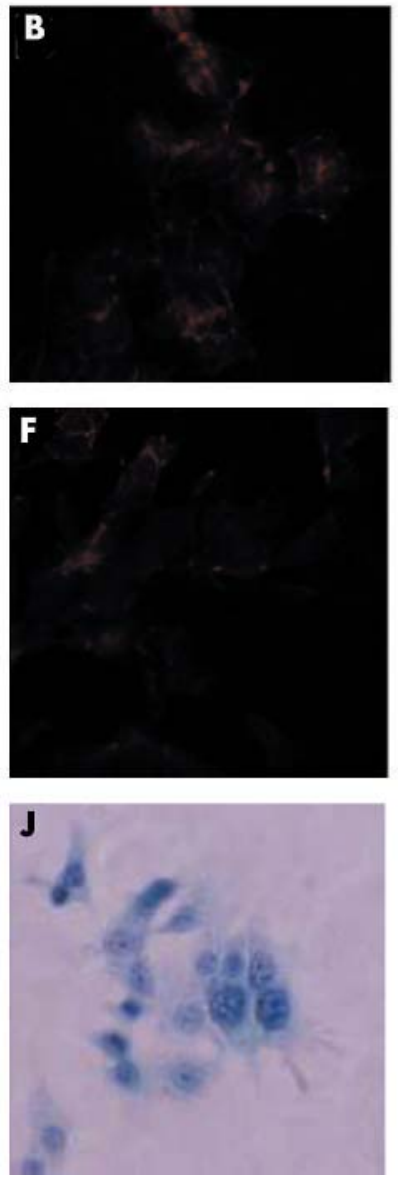

Capan-2 cells
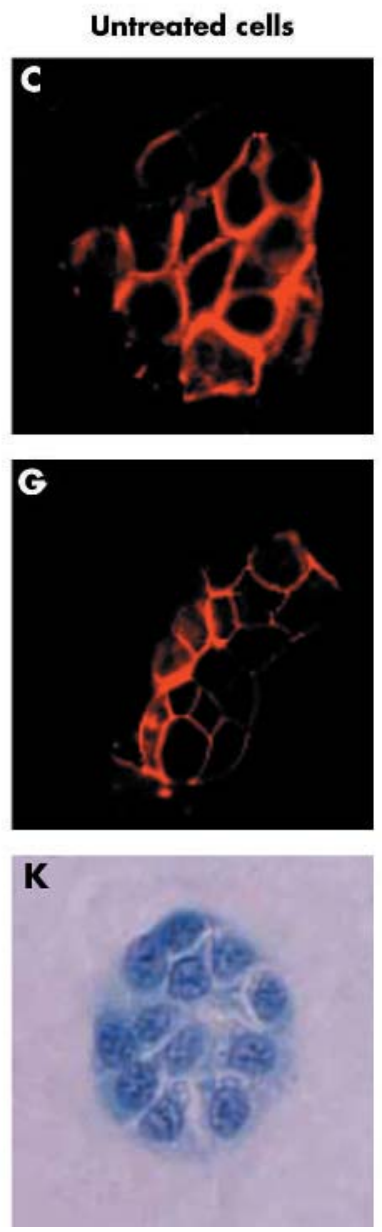

MMP-7 treatment
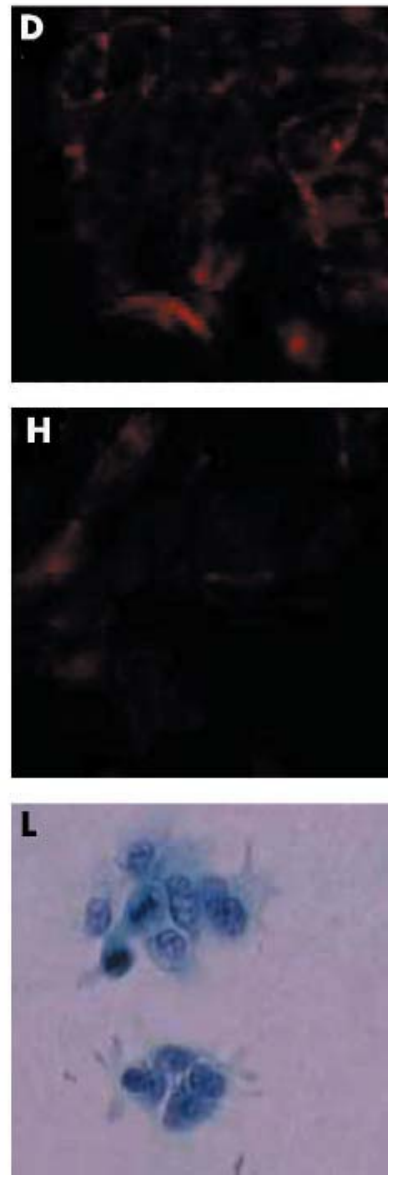

Figure 3 Disruption of tight junction (TJ) structures and translocations of TJ proteins induced by matrix metalloproteinase-7 (MMP-7) in nondissociated pancreatic cancer cells PC-1 and Capan-2. (A-H) Immunofluorescent staining; (I-L) Papanicolaou's staining. Original magnification, $\times 400$. ZO1, zonula occludens- 1 .

treatment with AG1478 or U0126 in all pancreatic cancer cell lines.

\section{MMP-7 induced the disruption of TJ structures and translocation of TJ proteins in non-dissociated pancreatic cancer cells}

The immunofluorescent staining images showed that occludin (fig 3A,3C) and ZO-1 (fig 3E,G) were exclusively localised to cell-cell junctions in untreated PC-1 and Capan-2 cells. However, after 36 hours of treatment with MMP-7, expression of occludin (fig 3B,D) and ZO-1 (fig 3F,H) was low at cell-cell junctions and they were both translocated to the cytoplasm in PC-1 and Capan-2 cells. In addition, the cell colonies of non-dissociated pancreatic cancer cells PC-1 (fig 3I) and Capan-2 (fig 3K) were disrupted by MMP-7 treatment (fig 3J,L).

MMP-7 induced activation of EGFR mediated MEK-ERK signal transduction in non-dissociated pancreatic cancer cells

In untreated PC-1 and Capan-2 cells, staining for p-EGFR (mean FI, 3.23 (SD, 0.26) and $2.48(0.18)$, respectively; fig 4A,B), p-MEK (mean FI, 4.62 (SD, 1.07) and 2.95 (0.47), respectively; fig 4E,F), and p-ERK (mean FI, 1.93 (SD, 0.46) and $2.15(0.38)$, respectively; fig $4 \mathrm{I}, \mathrm{J})$ was faint. In contrast, the expression of p-EGFR (mean FI, 56.76 (SD, 8.06) and 63.68 (14.82); $\mathrm{p}=0.008$ and $\mathrm{p}=0.019$, respectively; fig 4C,D), p-MEK (mean FI, 37.19 (SD, 4.66) and 30.30 (3.02); $p=0.004$ and $p=0.003$, respectively; fig $4 \mathrm{G}, \mathrm{H}$ ), and p-ERK (mean FI, 23.18 (SD, 2.39) and 20.11 (2.28); $\mathrm{p}=0.003$ and $\mathrm{p}=0.004$, respectively; fig $4 \mathrm{~K}, \mathrm{~L})$ was significantly induced by MMP-7 treatment in PC-1 and Capan-2 cells. Figure 4 parts $\mathrm{M}$ and $\mathrm{N}$ show the average FI values, respectively.

\section{In vitro invasiveness of pancreatic cancer cells}

As shown in fig 5A, non-dissociated pancreatic cancer cells PC-1 and Capan-2 were weakly invasive (mean number of invasive cells, 10.7 (SE, 3.1) and 4.7 (1.5), respectively). Surprisingly, the invasive capability was significantly enhanced by 12 hours of treatment with MMP-7 (mean number of invasive cells, 89.3 (SE, 14.0) and 38.3 (10.6); $\mathrm{p}=0.015$ and $\mathrm{p}=0.024$, respectively).

In contrast, as shown in fig 5B, dissociated cells PC-1.0 and AsPC-1 were strongly invasive (mean number of invasive cells, 31.3 (SE) 4.2 and 28.3 (4.5), respectively). However, their invasive ability was suppressed by AG1478 treatment (mean number of invasive cells, 8.3 (SE) 1.5 and 8.9 (SE) 2.5; $\mathrm{p}=0.005$ and $\mathrm{p}=0.004$, respectively) or U0126 treatment (mean number of invasive cells, 5.6 (SE) 1.5 and 9.3 (1.5); $\mathrm{p}=0.004$ and $\mathrm{p}=0.008$, respectively). 


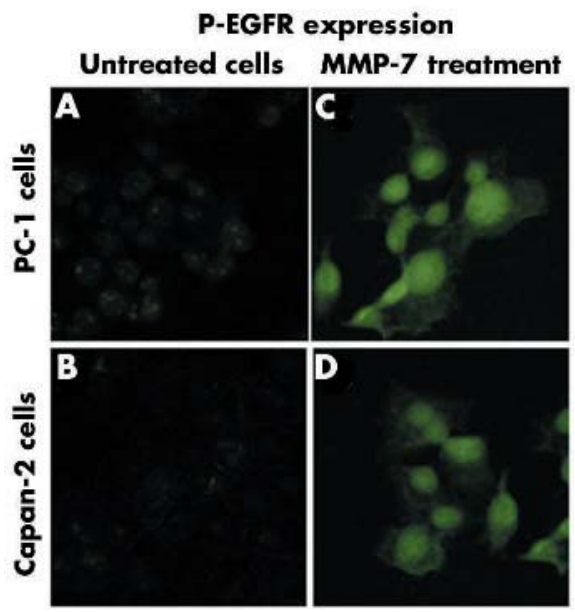

FI of signal molecules expression $M$ in PC-1 cells

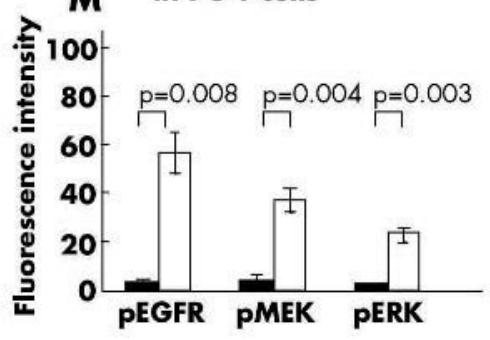

P-MEK expression Untreated cells
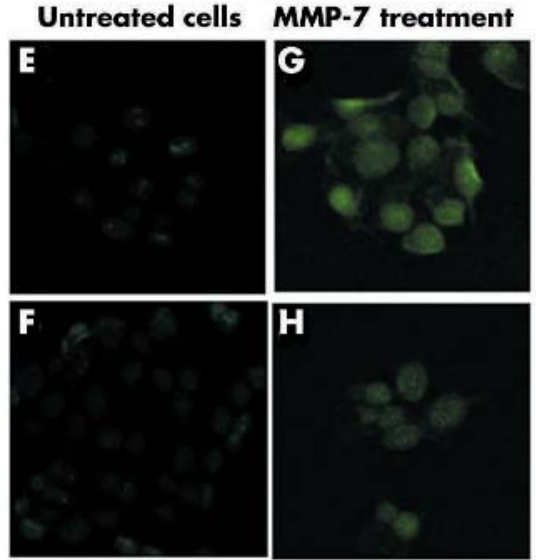

FI of signal molecules expression $N$ in Capan-2 cells

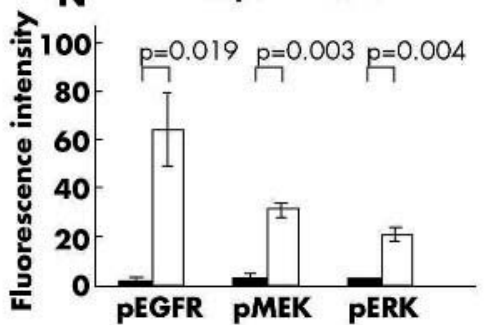

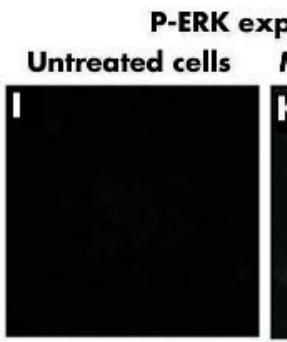
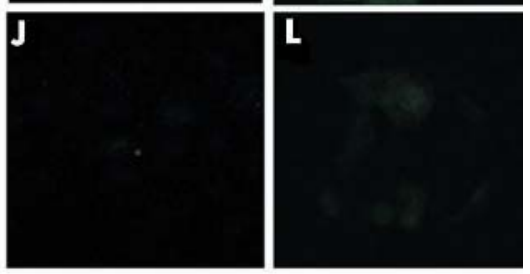

Figure 4 Activation of the epidermal growth factor receptor (EGFR) mediated mitogen activated protein kinase kinase (MEK)-ERK (extracellular signal regulated protein kinase) signalling pathway by matrix metalloproteinase-7 (MMP-7) treatment in non-dissociated pancreatic cancer cells PC-1 and Capan-2. Original magnification, $\times 400$. p, phosphorylated. Closed bars, untreated cells; open bars, MMP-7.

MMP-7 expression in human pancreatic cancer tissues No immunostaining for MMP-7 was seen in non-malignant pancreatic ductal/ductular cells (fig 6A), the cell type from which the pancreatic cancer cell lines used in our current study originated. In pancreatic cancer tissue, MMP-7 expression was seen both at the centre and at the invasive front of pancreatic tumours (fig 6B). Furthermore, MMP-7 expression was significantly stronger at the invasive front than at the centre of the same pancreatic cancer tissue.

\section{DISCUSSION}

The MEK-ERK signal transduction pathway is a key intracellular signalling pathway that is involved in diverse cellular activities, including cell proliferation, cell division, cell motility, apoptosis, and others. ${ }^{16}$ In our previous studies, we found that activation (phosphorylation) of the EGFR mediated MEK-ERK signalling pathway significantly induced cell dissociation in non-dissociated pancreatic cancer cells PC-1 and Capan-2. In contrast, inhibition of this signalling pathway by the EGFR inhibitor AG1478 or MEK inhibitor U0126 induced cell aggregation in dissociated pancreatic cancer cells PC-1.0 and AsPC-1. ${ }^{4-6}$ In our current study, the conditioned medium localised MMP-7 protein, not the intracellular localised proMMP-7 protein, showed a distinctly different pattern of expression between dissociated and non-dissociated pancreatic cancer cells. Furthermore, both the intracellular and conditioned medium localised MMP-7 proteins were suppressed by AG1478 or U0126 treatment. The invasiveness of non-dissociated cells was also found to be enhanced by MMP-7 treatment. In addition, the stronger expression of MMP-7 at the invasive front compared with the centre of human pancreatic tumours was consistent
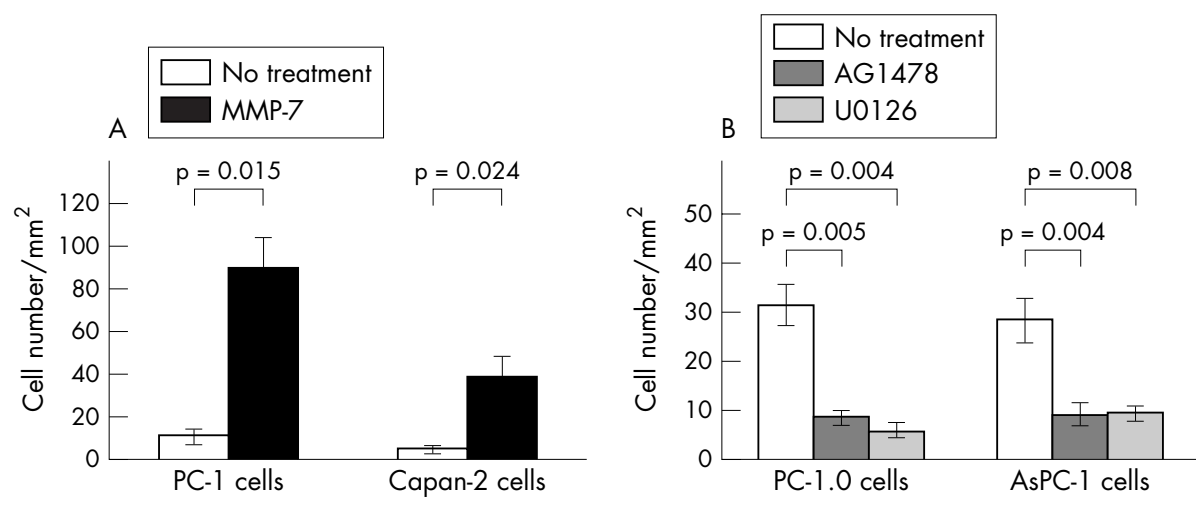

Figure 5 Chemoinvasion of pancreatic cancer cells. (A) Matrix metalloproteinase-7 (MMP-7) treatment greatly enhanced the chemoinvasion of non-dissociated cells PC-1 and Capan2. (B) AG1478 and U0126 treatment suppressed the chemoinvasion of dissociated cells PC-1.0 and AsPC-1. The invasive cell number is presented as mean (SE). All experiments were done in triplicate. 

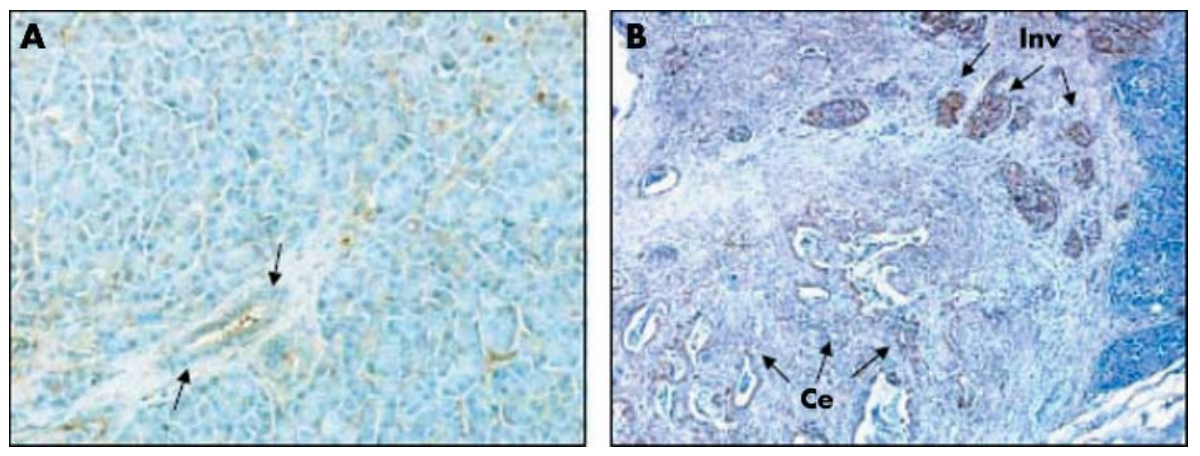

Figure 6 Immunohistochemical expression of matrix metalloproteinase7 (MMP-7) in human pancreatic cancer tissues. (A) The expression of MMP-7 in non-malignant pancreatic tissues. The arrows show that no MMP-7 expression was seen in the pancreatic ductal/ ductular cells. (B) The expression of MMP-7 in pancreatic cancer tissue. The arrows show that the expression of MMP-7 at the invasive front (Inv; upper right) is significantly stronger than at the centre (Ce; lower left) of the tumour. Original magnification, $\times 100$.

with the expression pattern of MEK2. ${ }^{4}$ These results imply that the MMP-7 protein is closely involved in cell dissociation and the subsequent invasion of pancreatic cancer cells. In addition, the EGFR mediated MEK-ERK signalling pathway may closely correlate with MMP-7 expression during cell dissociation and the subsequent invasion of pancreatic cancer cells.

\begin{abstract}
"Matrix metalloproteinase-7 may induce cell dissociation through the disruption of tight junctions and the translocation of tight junction proteins in pancreatic cancer cells"
\end{abstract}

TJs are known to form continuous circumferential intercellular contacts between epithelial cells and to create a regulated barrier to the movement of paracellular molecules. ${ }^{17}$ We previously found that the pattern of expression and the distribution of TJ proteins closely correlated with activation of the MEK-ERK signalling pathway during the dissociation of pancreatic cancer cells. ${ }^{7}$ Our current study demonstrated the disruption of TJ structures and the translocation of TJ proteins from cell-cell junctions to the cytoplasm after the addition of MMP-7 to the culture medium of non-dissociated cells PC-1 and Capan-2. These findings indicate that MMP-7 may induce cell dissociation through the disruption of TJ structures and the translocation of TJ proteins in pancreatic cancer cells.

Interestingly, we found that the addition of MMP-7 to the culture medium of non-dissociated pancreatic cancer cells PC-1 and Capan-2 induced the activation of the EGFR mediated MEK-ERK signalling pathway in these cells. There is evidence that MMP-7 can interact with several cell surface

\section{Take home messages}

- Matrix metalloproteinase-7 (MMP-7) is closely involved in cell dissociation and the subsequent invasion of pancreatic cancer cells

- MMP-7 can induce cell dissociation in pancreatic cancer through activating epidermal growth factor receptor (EGFR) mediated mitogen activated protein kinase kinase (MEK)-ERK (extracellular signal regulated protein kinase) signalling, and through inducing the disruption of tight junction (TJ) structures and the translocation of TJ proteins

- MMP-7 and EGFR mediated MEK-ERK signalling possibly form a positive feedback loop to induce and sustain cell dissociation, which is directly related to the high potential for invasion and metastasis seen in pancreatic cancer molecules and growth factors (for instance, E-cadherin, $\beta 4$ integrin, tumour necrosis factor $\alpha$, and Fas ligand), thereby promoting specific cell functions. ${ }^{18-20}$ Moreover, MMP-7 also forms a complex with cell surface protein CD44, which leads to shedding of cell surface localised heparin binding epidermal growth factor, which is one of the ligands of EGFR. $^{21}$ However, whether MMP-7 activates the EGFR mediated MEK-ERK signalling pathway via this mechanism, and the exact EGFR ligand released by MMP-7 are still unclear. Taken together, MMP-7 expression is closely correlated with the activation of EGFR mediated MEK-ERK signalling in the cell dissociation associated with the invasion and metastasis of pancreatic cancer. These molecules possibly form a positive feedback loop, which may facilitate cell dissociation and the subsequent invasion-metastasis process in pancreatic cancer.

In summary, MMP-7 is closely involved in cell dissociation and subsequent invasion of pancreatic cancer cells. MMP-7 can induce cell dissociation in pancreatic cancer through activating EGFR mediated MEK-ERK signalling, and through inducing the disruption of TJ structures and the translocation of TJ proteins. MMP-7 and the EGFR mediated MEK-ERK signalling pathway possibly form a positive feedback loop to induce and sustain cell dissociation, which is directly related to the high potential for invasion and metastasis in pancreatic cancer. Controlling the expression of MMP-7 will contribute to the development of a new clinical therapeutic method based on anti-invasion and antimetastasis in pancreatic cancer.

\section{Authors' affiliations}

X Tan, H Egami, M Abe, F Nozawa, M Hirota, M Ogawa, Department of Gastroenterological Surgery, Graduate School of Medical Sciences, Kumamoto University, Kumamoto 860-8556, Japan

\section{REFERENCES}

1 Torimura T, Ueno T, Kin M, et al. Autocrine motility factor enhances hepatoma cell invasion across the basement membrane through activation of $\beta 1$ integrins. Hepatology 2001;34:62-71.

2 Egami H, Takiyama Y, Cano M, et al. Establishment of hamster pancreatic ductal carcinoma cell line (PC-1) producing blood group-related antigens. Carcinogenesis 1989;10:861-9.

3 Egami H, Tomioka T, Tempero M, et al. Development of intrapancreatic transplantable model of pancreatic duct adenocarcinoma in Syrian golden hamsters. Am J Pathol 1991;138:557-61.

4 Tan X, Egami H, Kamohara H, et al. Involvement of the mitogen-activated protein kinase kinase 2 in the induction of cell dissociation in pancreatic cancer. Int J Oncol 2004;24:65-73.

5 Tan X, Egami H, Ishikawa S, et al. Relationship between the expression of extracellular signal-regulated kinase $1 / 2$ and the dissociation of pancreatic cancer cells: involvement of ERK1/2 in the dissociation status of cancer cells. Int J Oncol 2004;24:815-20.

6 Tan X, Egami H, Ishikawa S, et al. Relationship between activation of epidermal growth factor receptor and cell dissociation in pancreatic cancer. Int J Oncol 2004;25:1303-9.

7 Tan X, Tamori Y, Egami H, et al. Analysis of invasion-metastasis mechanism in pancreatic cancer: involvement of tight junction transmembrane protein 
occludin and MEK/ERK signal transduction pathway in cancer cell dissociation. Oncol Rep 2004;11:993-8

8 Tan X, Egami H, Ishikawa S, et al. Arrangement of expression and distribution of tight junction protein claudin-1 in cell dissociation of pancreatic cancer cells. Int J Oncol 2004;25:1567-74.

9 Tan X, Egami H, Ishikawa S, et al. Zonula occludens-1 (ZO-1) redistribution is involved in the regulation of cell dissociation in pancreatic cancer cells. Dig Dis Sci 2005;50:1402-9.

10 Yamamoto $\mathrm{H}$, Itoh $\mathrm{F}$, Adachi $\mathrm{Y}$, et al. Relation of enhanced secretion of active matrix metalloproteinases with tumour spread in human hepatocellular carcinoma. Gastroenterology 1997;1 12:1290-6.

11 Yamamoto $\mathrm{H}$, Itoh F, Iku S, et al. Expression of matrix metalloproteinases and tissue inhibitors of metalloproteinases in human pancreatic adenocarcinomas: clinicopathologic and prognostic significance of matrilysin expression. J Clin Oncol 2001;19:1118-27.

12 Zhu XF, Liu ZC, Xie BF, et al. EGFR tyrosine kinase inhibitor AG1478 inhibits cell proliferation and arrests cell cycle in nasopharyngeal carcinoma cells. Cancer Lett 2001; 169:27-32.

13 Favata MF, Horiuchi KY, Manos EJ. Identification of a novel inhibitor of mitogen-activated protein kinase kinase. J Biol Chem 1998;273:18623-32.
14 Seiler N, Schneider Y, Gosse F, et al. Polyploidisation of metastatic colon carcinoma cells by microtubule and tubulin interacting drugs: effect on proteolytic activity and invasiveness. Int $J$ Oncol 2004; 25:1039-48.

15 Kurizaki T, Egami H, Hirota $M$, et al. Characterization of cancer cell dissociation factor in a highly invasive pancreatic cancer cell line. Cancer 1995;75:1554-61.

16 Cobb MH. MAP kinase pathways. Prog Biophys Mol Biol 1999;71:479-500.

17 Farquhar MG, Palade GE. Junctional complexes in various epithelia. J Cell Biol 1963;17:375-412.

18 Noe V, Fingleton B, Jacobs K, et al. Release of an invasion promoter Ecadherin fragment by matrilysin and stromelysin-1. J Cell Sci 2001;114:111-18

19 Von Bredow DC, Nagle RB, Bowden GT, et al. Cleavage of beta 4 integrin by matrilysin. Exp Cell Res 1997:236:341-5.

20 Haro H, Crawford HC, Fingleton B, et al. Matrix metalloproteinase-7dependent release of tumor necrosis factor-alpha in a model of herniated disc resorption. J Clin Invest 2000;105:143-50.

21 Stamenkovic I. Matrix metalloproteinases in tumor invasion and metastasis. Cancer Biol 2000;10:415-33.

\section{BMJ Publishing Group}

Associate editors: Journal of Clinical Pathology

London

The fournal of Clinical Pathology wishes to appoint two Associate Editors to oversee the peer review and development of its Chemical Pathology and Molecular Pathology sections.

Closing date 28 Feb 2006

The Journal of Clinical Pathology, currently in its 60th year of publication, is a leading international journal covering all aspects of pathology. It is co-owned by the Association for Clinical Pathologists and the BMJ Publishing Group, a wholly owned subsidiary of the British Medical Association. The Journal of Clinical Pathology aims to encompass the remit of human pathology in its broadest sense, incorporating all fields and branches.

The successful applicant will be driven, dynamic, enthusiastic, willing to work in a team, share ideas, prepared to actively solicit papers/reviews, promote the journal worldwide and invite opinion shapers in their area to contribute to the journal. He/she will have a recognised track (publishing) record in their field and regular contact with colleagues around the world. He/she will be expected to increase the number of submissions to the journal in their field and to commission and accept at least 4 reviews on hot topics in their area each year. Capacity to devote 1-2 hours/day to the journal, willingness to attend policy meetings in London at least once a year and experience of online submission and peer review systems are essential. Full training on the journal's online peer review system will be provided. Previous editor/editorial board experience is desirable but not essential. Tenure of the associate editor position is usually for 1 year and may be extended to a further 2 years by mutual agreement. We offer a small honorarium, based on the number of manuscripts handled, which is paid quarterly.

To apply, please email your CV, a short treatise on why you think you would make a good associate editor, how you would accomplish the aims of the journal in general and the requirements of the Associate Editor position in particular, and provide suggestions on how you could help the Journal of Clinical Pathology to become the pre-eminent pathology journal, to the Editor: Professor Runjan Chetty, Director of Surgical Pathology, University of Toronto

The RMlJ is an equal opportunities emp oyer and its policy has been ceveloped to ensure that $3 \mathrm{M}$. members and staff are fully atware ol cur comrriltrent to provide equality of opporturity ir all of cur functiers.
(Runjan.Chetty@uhn.on.ca; tel: 001-416-946-2876).

Closing date for applications: 28 February 2006.
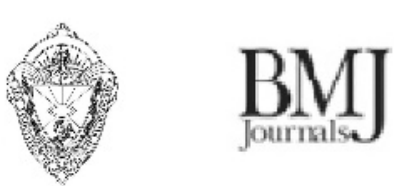